\title{
ESTUDIO DE COMPUESTOS VEGETALES CON POTENCIAL ACCIÓN ANTIFÚNGICA SOBRE PATÓGENOS DE PLANTAS CULTIVADAS
}

\author{
STUDY OF PLANT ORIGIN COMPOUNDS WITH POTENTIAL \\ ANTIFUNGAL ACTION AGAINST PATHOGENS OF CULTIVATED \\ PLANTS
}

\author{
Paula Badaracco ${ }^{1 *}$, Maximiliano Sortino ${ }^{2}$, Rosanna N. Pioli ${ }^{1,3,4}$ \\ ${ }^{1}$ Botánica Criptogámica y Laboratorio Biodiversidad Vegetal y Microbiana, Facultad de Ciencias \\ Agrarias, Universidad Nacional Rosario, Campo Experimental José Villarino, C.C. 14 (S2125ZAA), \\ Zavalla, Argentina. \\ ${ }^{2}$ Farmacognosia, Facultad de Ciencias Bioquímicas y Farmacia, Universidad Nacional de Rosario, \\ Suipacha 531 (S2002LRK), Rosario, Argentina. msortino@fbioyf.unr.edu.ar \\ ${ }^{3}$ Instituto de Investigaciones en Ciencias Agrarias de Rosario (IICAR, CONICET-Universidad Nacional \\ de Rosario), Campo Experimental José Villarino, C.C. 14 (S2125ZAA) Zavalla, Santa Fe, Argentina. \\ ${ }^{4}$ Consejo de Investigaciones de la Universidad Nacional de Rosario, Maipú 1065 (2000EKF) Rosario, \\ Santa Fe, Argentina. pioli@iicar-conicet.gob.ar \\ *Autor para correspondencia E-mail: paula.badaracco93@gmail.com
}

\section{RESUMEN}

Los hongos patógenos generan enfermedades en distintos cultivos y afectan el rendimiento y producción de alimentos. El control mediante fitosanitarios puede implicar un riesgo para la salud humana y el ambiente. En este contexto la utilización de los metabolitos vegetales como potenciales bio-controles de patógenos son una alternativa sustentable. El objetivo de este estudio fue evaluar la acción antifúngica de seis compuestos de origen vegetal, daidzeína, genisteína, dos chalconas, vainillina e iso-vainillina, sobre el crecimiento in vitro de patógenos que actúan en cultivos de interés alimentario. Se evaluó la Concentración Inhibitoria Mínima (CIM) y la Concentración Fungicida Mínima (CFM) de cada compuesto químico mediante el método de microdilución en caldo frente a cinco géneros fúngicos: Alternaria sp., Cladosporium sp., Fusarium (F. graminearum, F. subglutinans, F. proliferatum, F. oxysporum), Phomopsis longicolla, y Rhizopus stolonifer. Los resultados indicaron que daidzeína, genisteína, iso-vainillina y $\mathrm{CH1}$ no presentaron acción inhibitoria y/o fungicida en concentraciones menores a $250 \mu \mathrm{g} \mathrm{mL}{ }^{-1}$. $\mathrm{CH} 2$ se caracterizó por inhibir a cuatro aislamientos pertenecientes a tres géneros en diferentes concentraciones y presentar actividad fungicida sobre dos de los mismos. La vainillina demostró ser efectiva para inhibir y/o eliminar la totalidad de los aislamientos (nueve) incluidos en los cinco géneros fúngicos evaluados con concentraciones menores a $250 \mu \mathrm{g} \mathrm{mL}^{-1}$. Se concluyó que los metabolitos de origen vegetal como la vainillina y $\mathrm{CH} 2$ presentaron capacidad antifúngica en diferente grado y especificidad sobre los aislamientos.

Palabras clave: Metabolitos vegetales, hongos fitopatógenos, acción antifúngica

\section{ABSTRACT}

Pathogenic fungi generate crop diseases, and affect yield and food production. The use of phytosanitary products to control fungal diseases can pose a risk to human health and the environment. In this context, the use of plant metabolites as potential bio-controls for pathogens turns out to be a sustainable alternative. The objective of this study was to evaluate the anti-fungal 
action of six compounds of plant origin: daidzein, genistein, two chalcones, vanillin and isovanillin, on the in vitro growth of pathogens. The Minimum Inhibitory Concentration (MIC) and the Minimum Fungicidal Concentration (CFM) of each chemical compound were evaluated by the broth micro-dilution method against five fungal genera: Alternaria sp., Cladosporium sp., Fusarium (F. graminearum, F. subglutinans, F. proliferatum, F. oxysporum), Phomopsis longicolla, and Rhizopus stolonifer. The results indicated that daidzein, genistein, iso-vanillin and CH1 did not show an inhibitory and / or fungicidal action at concentrations lower that $250 \mu \mathrm{g} \mathrm{mL} \mathrm{L}^{-1}$. CH2 was characterized by inhibiting four isolates belonging to three genera in different concentrations, and presenting fungicidal activity on two of them. Vanillin proved to be effective to inhibit and / or eliminate all of the isolates (nine) included in the five fungal genera evaluated at concentrations below $250 \mu \mathrm{g}$ $\mathrm{mL}^{-1}$. It is concluded that metabolites of plant origin such as vanillin and $\mathrm{CH} 2$ presented antifungal capacity in different degrees and specificity on the isolates.

Key words: Plant metabolites, phytopathogenic fungi, antifungal action

\section{INTRODUCCIÓN}

Las plantas, a lo largo del tiempo, han debido adaptarse a factores bióticos y abióticos, y han desarrollado estrategias físicas, químicas y fisiológicas, para poder hacer frente a todo aquello que tienda a afectar su normal desarrollo (Taiz y Zeiger, 2006).

Dentro de los factores bióticos se encuentran los hongos. La diversidad fúngica es la resultante de diversas estrategias y mecanismos de variabilidad genética y reproductiva (Agrios, 2005). Dentro de este Reino, los hongos fitopatógenos son capaces de desarrollar enfermedades en distintos hospedantes y momentos de su ciclo biológico (Alexopoulos et al., 1996; Newton et al., 2010). La importancia de controlarlos reside en que las patologías que generan no sólo afectan a la producción agrícola, reduciendo la calidad de los alimentos y la viabilidad de las semillas, sino que además suelen ser nocivas para la salud humana y animal (Yanar et al., 2011).

El proceso patogénico fúngico resultará efectivo cuando las esporas germinen y sean capaces de superar las barreras defensivas vegetales que pueden ser físicas y/o químicas. Dentro de estas últimas, las plantas poseen una variedad de extractos y compuestos biológicamente activos que expresan acción antifúngica (Ali et al., 2017). Dicha actividad antimicrobiana es generada por metabolitos secundarios, como son los compuestos fenólicos, saponinas, alcaloides, terpenoides, estibilenos y fenilpropanoides, denominados anticipinas (Iriti y Varoni, 2017).

Estos metabolitos surgen como una primera línea de defensa vegetal ya sea inhibiendo al microorganismo o disminuyendo la colonización, para que luego otras formas de defensa puedan controlar su desarrollo (Lygin et al., 2014). De acuerdo a Rodríguez-Maturino et al. (2015), tales compuestos participan en el proceso defensivo vegetal, con la ventaja adicional de ser rápidamente degradados en el suelo y no presentar (generalmente) un efecto tóxico en mamíferos. En consecuencia, podrían ser empleados como biocontroladores de patógenos en los sistemas de agricultura orgánica y sustentable.

Distintas familias botánicas producen compuestos con acción antifúngica, los cuales pueden variar en su constitución química dentro de cada familia o bien estar presentes parcialmente dentro de las mismas (Taiz y Zeiger, 2006). De acuerdo a Cesco et al. (2010), los metabolitos derivados de fenilpropanoides están ampliamente distribuidos en el reino vegetal y desempeñan distintas funciones. De allí la importancia de analizar este tipo de compuestos, donde su biosíntesis puede ser la respuesta inducida a la presencia de un patógeno fúngico.

En la Familia Fabaceae, según Lygin et al. (2013), los fenilpropanoides son sintetizados y acumulados como respuesta al ataque de patógenos. En Glycine max se sintetizan isoflavonas como ser daidzeína, genisteína y sus derivados; pero además isoflavonoides como coumestrol y gliceolina (Graham, 1991; Lozovaya et al., 2004; Lygin et al., 2013). Más allá de esta especie en particular, otras dentro de esta familia botánica presentan compuestos químicos con potenciales funciones antifúngicas. Por lo tanto el análisis de diversos compuestos producidos por diferentes géneros de leguminosas permitiría un mejor conocimiento sobre la defensa frente a los patógenos por parte de dicha familia.

Carpinella et al. (2003) mencionaron que es necesario prestar atención a la familia Meliaceae, debido a que ciertos metabolitos potencialmente activos son producidos por árboles de dicha familia, los cuales pueden ser utilizados como compuestos antifúngicos. Particularmente Melia azedarach, conocido vulgarmente como paraíso, produce ciertos metabolitos dentro de los compuestos vegetales que limitan el desarrollo 
de patógenos fúngicos.

Más allá de un grupo particular de familias botánicas, la presencia de metabolitos secundarios dentro del Reino Vegetal muestra que los mismos proponen distintas formas de contención a la infección y/o colonización de ciertos patógenos. El conocimiento de la actividad de estos metabolitos vegetales con efectos de menor impacto ambiental, hicieron que fuesen considerados como una estrategia natural para controlar aquellos patógenos fúngicos asociados a los alimentos y que ponen en riesgo la salud humana y animal (Yanar et al., 2011).

En dicho contexto, el objetivo de este trabajo fue determinar la acción inhibitoria de seis metabolitos de origen vegetal: daidzeína, genisteína, dos chalconas ( $\mathrm{CH} 1$ y CH2), vainillina ( 3 metoxi- 4 hidroxi benzaldehído) e iso-vainillina (4 metoxi-3 hidroxi-benzaldehído) sobre el crecimiento in vitro de patógenos que actúan en cultivos de interés alimentario.

\section{MATERIALES Y MÉTODOS}

\section{Selección y aislamiento de cepas fúngicas a ser evaluadas}

Los aislamientos fúngicos evaluados pertenecen a cinco géneros taxonómicos. Cuatros cepas de Fusarium y una de Phomopsis fueron seleccionadas a partir de la micoteca del área Fitopatología, disponible en el Laboratorio de Biodiversidad Vegetal y Microbiana (BioVyM,) y el Instituto Mixto UNR-CONICET (IICAR), de la Facultad Ciencias Agrarias de la Universidad Nacional de Rosario. Estos patógenos fúngicos fueron reactivados mediante siembras en medio de cultivo de Agar-Papa-Glucosa $2 \%$ y acidulado con gotas de ácido láctico al 25\% (APGA), e incubados en condiciones de oscuridad a $26+/-1^{\circ} \mathrm{C}$ durante 7 días hasta la obtención de colonias.

A dicha selección se incorporaron cuatro nuevos aislamientos patogénicos de especies hortícolas de consumo humano, obtenidos de frutos del cultivo de Cucurbita máxima var. maxima (zapallito). Para ello se procedió a tomar porciones de tejido del área de avance de las lesiones y se desinfectaron superficialmente con hipoclorito de sodio al $1 \%$ durante 30 segundos. Se realizaron lavados con agua estéril, luego se orearon y sembraron en placas con medio de cultivo APGA. Se incubaron en condiciones de oscuridad a $26+/-1^{\circ} \mathrm{C}$ durante 7 días hasta la obtención de colonias.

\section{Caracterización morfológica de las especies fúngicas evaluadas}

Una vez obtenidas las colonias puras de cada uno de los patógenos fúngicos en estudio, se realizó la caracterización de cada aislamiento en base a atributos o marcadores morfológicos con la finalidad de validar su identidad. Fueron evaluadas las características macromorfológicas (color de las colonias, presencia y forma del estroma, presencia y tipo de picnidios $\mathrm{y}$ peritecios) y micro-morfológicas (tamaño $\mathrm{y}$ forma de conidios, ascos y ascosporas) (Barnett y Hunter, 1998; Pioli et al., 2000; 2004; Leslie y Summerell, 2006; Hernández et al., 2015). Además algunas cepas fúngicas fueron identificadas en base a dos técnicas moleculares basadas en la reacción en cadena de la polimerasa (PCR) y uso de oligonucleóticos (primers) de diferente especificidad (Pioli et al., 2003; Udayanga et al., 2012; Hernández et al., 2015).

\section{Preparación de inóculos}

Los inóculos se prepararon como suspensiones de conidios de acuerdo al protocolo M38-A2 recomendado por el Instituto Standard de Clínica y Laboratorio (CLSI) de Estados Unidos (CSLI, 2008). Los hongos fueron sembrados en medio Agar-Papa-Dextrosa e incubados a $28^{\circ} \mathrm{C}+$-durante 10 días para estimular la conidiación. Una vez obtenidas colonias maduras, las mismas se cubrieron con agua destilada estéril y se rasparon para obtener una suspensión densa. La solución se agitó por medio de un vortex por 3-5 minutos. Posteriormente esta suspensión se dejó reposar por 5 a 7 min para permitir la sedimentación de los fragmentos más pesados y se separó la fracción superior. Esta última fracción se homogeneizó y se realizó el recuento de conidios en cámara de Neubauer (Svetaz et al., 2004). Se realizaron los ajustes necesarios para obtener una concentración de $5 \times 10^{3} \mathrm{UFC} \mathrm{mL}^{-1}$ (Leslie and Summerell, 2006).

\section{Compuestos a evaluar}

Se trabajó con compuestos producidos por diferentes familias botánicas, entre ellas, la familia Meliaceae y Fabaceae (Graham, 1991; Carpinella et al., 2003; Pioli et al., 2004) pero sintetizados por el Laboratorio Sigma- Aldrich. Los compuestos de origen vegetales a evaluar fueron: daidzeína y genisteína (Lozovaya et al., 2004; Pioli et al., 2004), vainillina: 3 metoxi- 4 hidroxi benzaldehído (Carpinella et al., 2003). Además se evaluó la actividad antifúngica de compuestos sintéticos y comerciales como iso-vainillina (4 metoxi- 3 hidroxi- benzaldehído), chalcona 1 (CH1) $\left(2^{\prime}, 3^{\prime}, 4^{\prime}\right.$ trimetoxi-1,3- difenil- 2 propen- 1 ona) y chalcona 2 (CH2) (1,3- difenil- 2 propen- 1 ona) (Fig. 1).

Además se utilizaron como testigos dos fungicidas comerciales cuyos principios activos son:a) azoxy trobina (estrobirulina)+ciproconazole (triazol), y b) carbendazim (2-metoxicarbamoilbencimidazol), ambos de baja toxicidad (grado 3 Banda azul o Banda verde) por ser catalogados 
<smiles>O=c1c(-c2ccc(O)cc2)coc2cc(O)ccc12</smiles>

daidzeína<smiles>O=c1c(-c2ccc(O)cc2)coc2cc(O)cc(O)c12</smiles>

genisteína<smiles>COc1cc(C=O)ccc1O</smiles>

vainillina<smiles>COc1ccc(C=O)cc1O</smiles>

isovainillina<smiles>COc1cc(C(=O)/C=C/c2ccccc2)cc(OC)c1OC</smiles>

chalcona 1<smiles>O=C(/C=C/c1ccccc1)c1ccccc1</smiles>

chalcona 2

Fig. 1. Estructura química de compuestos evaluados (Graficadas con el programa Chem Bio Draw Ultra $\mathbf{1 2 . 0}$

Fig. 1. Chemical structure of the evaluated compounds (Drawn using the Chem Bio Draw Ultra 12.0 software)

los menos tóxicos para la salud humana y el ambiente (INTA, 2008).

Para realizar el análisis, los distintos compuestos químicos puros fueron disueltos en solvente DMSO para lograr una concentración de $12,5 \mathrm{mg} \mathrm{mL}^{-1}$ (Zacchino y Gupta, 2007). Con respecto a los productos comerciales, los mismos fueron disueltos en agua debido a su mayor solubilidad en la misma (Zacchino y Gupta, 2007). De esta manera se obtuvieron las distintas soluciones madres de trabajo.

\section{Actividad antifúngica}

La actividad antifúngica fue determinada mediante la Concentración Inhibitoria Mínima (CIM) y la Concentración Fungicida Minina (CFM). La CIM se define como la menor concentración de un compuesto en la que se inhibe totalmente el desarrollo de las colonias de microorganismos fúngicos. En el caso de compuestos puros se consideraron activos aquellos que registran valores de CIM $<250 \mu \mathrm{g}$ $\mathrm{mL}^{-1}$ (Svetaz et al., 2004; Zacchino y Gupta, 2007; Sortino et al., 2011). La CIM de cada compuesto químico se determinó usando el método de micro-dilución en caldo en microplacas de 96 pocillos donde el medio utilizado fue SabouraudGlucosa (Sb-Glu).

A partir de las soluciones madres de los distintos compuestos a evaluar, se prepararon soluciones de trabajo en medio de cultivo Sb-Glu. En las microplacas se procedió a realizar diluciones seriadas para obtener diferentes concentraciones entre $250 ; 125 ; 62,5 ; 31,3 ; 15,6 ; 7,80 ; 3,90 ; 1,95 ; 0,98$; 0,49; $0,24 \mu \mathrm{g} \mathrm{mL} \mathrm{mL}^{-1}$ de los mismos. Cada uno de los pocillos presentó un volumen final de $100 \mu \mathrm{L}$ y se realizaron dos repeticiones por cada compuesto evaluado (Sortino et al., 2011). Posteriormente, se agregó $100 \mu \mathrm{L}$ de suspensión de inóculo de cada una de las especies fúngicas con el fin de evaluar el accionar del compuesto sobre las mismas. Previamente, se realizó un control de viabilidad del hongo colocando al mismo en medio de cultivo libre de los compuestos a evaluar. Las placas se incubaron a $28-30^{\circ} \mathrm{C}$ utilizando cámara húmeda por un tiempo de 2 a 5 días dependiendo de la especie fúngica.

Además se determinó la CFM, que se define como la mínima concentración del compuesto antifúngico necesario para provocar la muerte (efecto fungicida) de la mayoría de los microorganismos fúngicos viables (Zacchino y Gupta, 2007). Para ello, se tomaron alícuotas de 10 $\mu \mathrm{L}$ de aquellos pocillos en los que no se observó desarrollo fúngico y se sembraron en placas de Petri con Sb-Agar. Luego de la incubación por 2-5 días a $28^{\circ} \mathrm{C}$, se observó el crecimiento y se determinó la CFM.

\section{RESULTADOS Y DISCUSIÓN}

Caracterización morfológica de especies fúngicas evaluadas

Cada una de las especies fúngicas evaluadas 
presentó las características típicas descriptas bibliográficamente (Barnett y Hunter, 1998; Leslie y Summerell, 2006). Se determinó la identidad de aislamientos de los siguientes grupos taxonómicos: Cladosporium sp., Alternaria sp., Rhizopus stolonifer; Phomopsis longicolla, Fusarium graminearum CE-135, Fusarium graminearum CE170, Fusarium subglutinans, Fusarium proliferatum y Fusarium oxysporum. La validación de identidad y diferenciación entre cepas fue corroborada por métodos moleculares (Hernandéz et al., 2015).

\section{Compuestos evaluados y actividad antifúngica}

En base a los resultados obtenidos, los compuestos tanto naturales como sintéticos presentaron respuestas diferentes frente a los patógenos bajo estudio. Dos de los seis compuestos evaluados, la vainillina y $\mathrm{CH} 2$, mostraron efecto inhibidor $\left(\mathrm{CIM}<250 \mu \mathrm{g} \mathrm{mL} \mathrm{mL}^{-1}\right)$ y/o fungicida (CFM $<250 \mu \mathrm{g} \mathrm{mL}^{-1}$ ) frente a una parte o la totalidad de los nueve aislamientos fúngicos evaluados (Tabla 1).

Coincidiendo con Carpinella et al. (2003), la vainillina presentó actividad inhibitoria (CIM $=15,6$ a $\left.62,5 \mu \mathrm{g} \mathrm{mL}^{-1}\right)$ y fungicida $(\mathrm{CFM}=31,3 \mathrm{a}$ $\left.62,5 \mu \mathrm{g} \mathrm{mL} \mathrm{mL}^{-1}\right)$ efectivas frente a los 9 aislamientos evaluados (Tabla 1). Estos resultados demostraron que la vainillina es capaz de inhibir el crecimiento de colonias fúngicas, pero resulta más relevante aun su accionar fungicida, debido a que evitó o eliminó la posibilidad de crecimiento de los micelios evaluados. El rol y efecto de éste y otros compuestos semejantes son importantes de analizar debido a la eficacia potencial sobre el control sustentable de enfermedades en las producciones agrícolas y hortícolas. Además, como lo plantea Carpinella et al. (2003), su presencia en los alimentos como saborizante indicaría su baja toxicidad para los mamíferos, lo cual le brinda mayores beneficios en la obtención de materia prima agrícola y subproductos derivados alimenticios.

La iso-vainillina, isómero sintético dela vainillina, contrariamente no presentó acción inhibitoria en el desarrollo de las colonias fúngicas de las cepas estudiadas, excepto en el caso de Alternaria sp., a la cual inhibió en una concentración de CIM = $250 \mu \mathrm{g} \mathrm{mL} \mathrm{m}^{-1}$. Asimismo, no registró acción bio-fungicida tal lo demuestran los valores de CFM > $250 \mu \mathrm{g} \mathrm{mL}^{-1}$ (Tabla 1).

Las chalconas, de origen natural pero también sintético, presentaron comportamientos diferentes. $\mathrm{CH} 2$ se caracterizó por inhibir a Alternaria sp., P. longicolla, F. proliferatum y $F$. subglutinans en concentraciones de CIM $=62,5$ a $125 \mu \mathrm{g} \mathrm{mL}-1$ (Tabla 1). Respecto a su actividad fungicida, este compuesto sólo fue efectivo sobre Alternaria sp. y P. longicolla en concentraciones de
CFM de 125 y $250 \mu \mathrm{g} \mathrm{mL}^{-1}$, respectivamente. CH1, por su parte, no desarrolló actividad inhibitoria ni fungicida para los hongos analizados (Tabla 1).

Al comparar estos resultados con los de Svetaz et al. (2004), se pudo observar que las chalconas, como grupo químico, tienen distintas respuestas frente a diferentes patógenos o cepas de un mismo patógeno. Esto quedó demostrado al analizar los resultados obtenidos en este trabajo, donde si bien algunos efectos pueden ser similares, los valores de CIM y CFM no son equivalentes a los presentados por Svetaz et al. (2004), incluso al analizar el comportamiento frente a las dos cepas de $F$. graminearum que fueron evaluadas en ambos trabajos. Tal vez la efectividad de estas moléculas se deba al origen y/o composición química de dichos compuestos. Aquellos planteados por Svetaz et al. (2004) son extraídos de la parte aérea de Zuccagnia punctata (Fabaceae, Caesalpinoideae, Caesalpinieae) mientras que las chalconas evaluadas en este trabajo son de origen sintético. Esto podría explicar que su accionar aun frente al mismo patógeno sea diferente.

Por su parte, daidzeína y genisteína no mostraron acción inhibitoria ni fungicida sobre las cepas fúngicas en estudio en ninguna de las concentraciones estudiadas (CIM y CFM> 250 $\mu \mathrm{g} \mathrm{mL} \mathrm{m}^{-1}$ ) (Tabla 1). Por lo tanto estos metabolitos vegetales provenientes de la familia Fabaceae no han controlado la formación de colonias de los géneros y especies fúngicas evaluados bajo estas condiciones experimentales. Estos resultados fueron similares a los obtenidos in vivo (G. max) e in vitro por Pioli et al. (2004) sobre dos especies de Phomopsis, tales como P. longicolla y P. phaseoli var. meridionalis y var. caulivora, pero se contrapone a los resultados obtenidos por Lozovaya et al. (1999), donde dichos compuestos fueron efectivos in vivo frente a patógenos de infección radicular como algunas cepas de Fusarium causales de la muerte repentina o súbita en soja en EE.UU. Tal vez se debería considerar, como lo expresa Pioli et al. (2004), que la concentración y efectividad de estos isoflavonoides frente a la infección dependerá no sólo del patógeno al cual se enfrenta, sino además del tiempo transcurrido a partir de la penetración, y la distribución diferencial de estos compuestos a través de los distintos tejidos de la planta infectada.

Respecto a los fungicidas comerciales, incluidos como controles experimentales y considerados de baja toxicidad por las entidades reguladoras, se pudo observar que los principios activos tienen un accionar diferencial frente a los patógenos bajo estudio, pudiendo ser efectivos para inhibir y/o evitar el crecimiento de algunas cepas fúngicas con valores mínimos de CIM y 


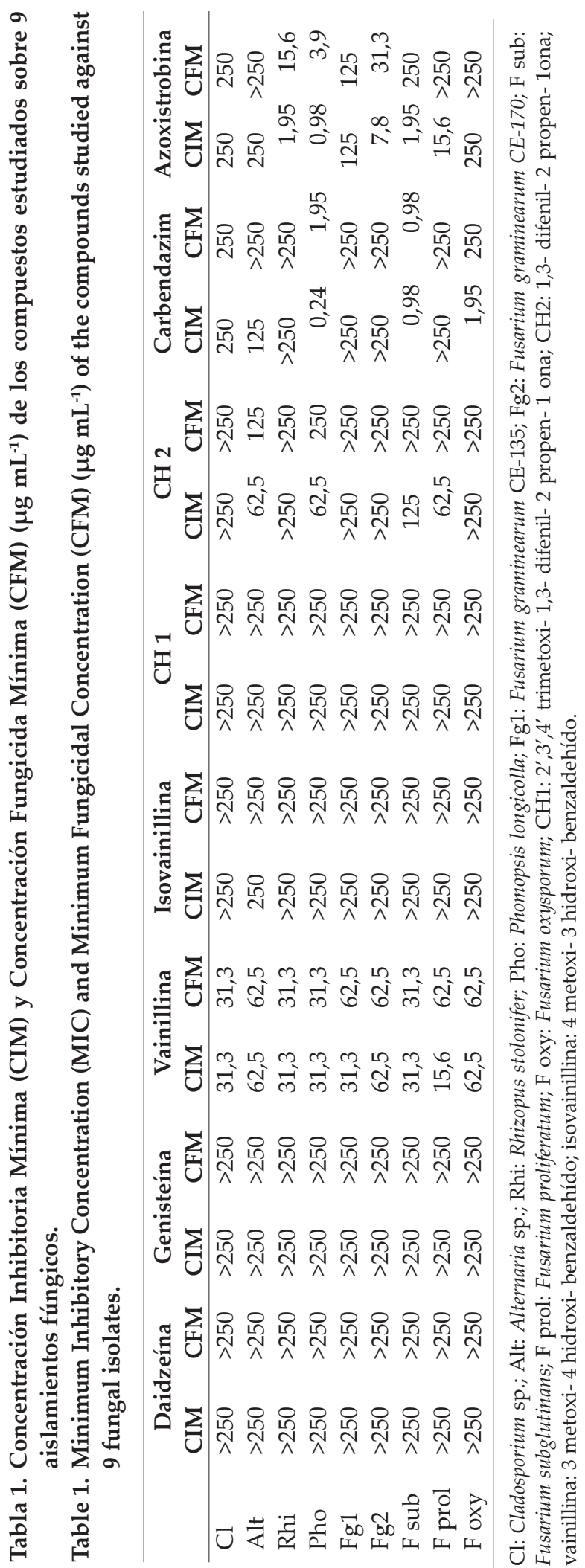


CFM $\left(<5 \mu \mathrm{g} \mathrm{mL}^{-1}\right)$ o bien no controlando a otras.

El carbendazim ha tenido un accionar inhibitorio variable frente a las distintas especies. El mismo fue capaz de inhibir de manera contundente el crecimiento de $P$. longicolla, $F$. subglutinans y F. oxysporum con valores de CIM $<2 \mu \mathrm{g} \mathrm{mL}{ }^{-1}$, pero no fue efectivo para controlar el crecimiento de las cepas de $F$. graminearum, F. proliferatum y R. stolonifer (CIM $>250 \mu \mathrm{g} \mathrm{mL}$ $\left.{ }^{1}\right)$ (Tabla 1). Al analizar la capacidad fungicida, este compuesto obtuvo valores de CFM similares a los respectivos CIM para $P$. longicolla y $F$. subglutinans, mientras que la CFM fue de $250 \mu \mathrm{g}$ $\mathrm{mL}^{-1}$ para F. oxysporum (Tabla 1).

El hecho de que no se encontró actividad inhibitoria ni fungicida del carbendazim frente a $R$. stolonifer, coincide con lo expresado por Cavallo et al. (2005). Asimismo, dicho principio activo no ha podido inhibir y/o presentar efecto fungicida sobre las cepas de $F$. graminearum CE-170 y CE-135 (CIM y CFM > $250 \mu \mathrm{g} \mathrm{mL}^{-1}$ ) diferenciándose de los resultados obtenidos por Svetaz et al. (2004).

La azoxistrobina inhibió el crecimiento fúngico en concentraciones disímiles variando desde una CIM de $250 \mu \mathrm{g} \mathrm{mL}^{-1}$ para Cladosporium sp., Alternaria sp. y F. oxysporum, a valores de CIM que variaron entre 0,98 y $125 \mu \mathrm{g} \mathrm{mL}^{-1}$ frente a las otras seis cepas fúngicas bajo estudio (Tabla 1).

Particularmente el género Fusarium presentó diferencias evidentes de acción antifúngica sobre las distintas especies, y también al comparar los resultados obtenidos en este trabajo y los publicados por otros autores. Esto sucede con F. oxysporum que fue controlado en valores limites por el compuesto activo de azoxistrobina, resultados que coinciden con lo planteado por Araya (2013). Asimismo al comparar el efecto ejercido sobre las dos cepas de F. graminearum evaluadas, se obtuvieron respuestas disímiles a otros autores, ya que las concentraciones inhibitorias y/o fungicidas fueron mayores a las presentadas por Svetaz et al. (2004).

En este contexto es oportuno destacar, además, que la azoxistrobina frente a $R$. stolonifer, $P$. longicolla y la cepa CE-170 de F. graminearum, registró efecto fungicida con valores de CFM = 15,6- 31,3 $\mu \mathrm{g} \mathrm{mL}-1$. Pero además se obtuvieron valores semejantes de CIM y CFM para la cepa CE-135 de F. graminearum y Cladosporium sp. (Tabla 1). Adicionalmente, la azoxistrobina fue capaz de inhibir el crecimiento de colonias de Alternaria sp., F. proliferatum y F. oxysporum en diferente grado, pero sin ejercer acción fungicida $\left(\mathrm{CFM}>250 \mu \mathrm{g} \mathrm{mL}^{-1}\right)$ sobre ellas.

Los resultados demuestran que la efectividad de la vainillina y $\mathrm{CH} 2$ fueron semejantes y/o hasta superiores a la expresada por los compuestos comerciales.

Considerando que ciertos patógenos no sólo generan problemas económicos y productivos, sino riesgos de calidad y sanidad alimentaria debido a las micotoxinas producidas y su accionar toxicogénico (Desjardins 2006; Peruzzo y Pioli, 2017), la importancia de este estudio reside en haber logrado identificar compuestos biodegradables de origen vegetal con acción biocontroladora sobre patógenos fúngicos de interés agronómico (Yanar et al., 2011).

La posterior evaluación y determinación experimental de las concentraciones y frecuencia de aplicación, acordes a la normativa vigente, brindará una opción de control sustentable de enfermedades vegetales, e inocuo para la salud humana, animal y el ambiente.

\section{CONCLUSIONES}

La vainillina y la chalcona $2(\mathrm{CH} 2)$ mostraron acción antifúngica en diferente grado $\mathrm{y}$ especificidad sobre los aislamientos evaluados.

El desarrollo in vitro de los aislamientos de Alternaria sp., $R$. stolonifer, P. longicolla, F. graminearum, F. subglutinans, F. proliferatum, y F. oxysporum, obtenidos a partir de cultivos de interés alimentario, fue inhibido al menos por dos de los seis compuestos de origen vegetal evaluados.

La vainillina inhibió el crecimiento y/o actuó como bio-fungicida de las nueve cepas evaluadas, pertenecientes a ocho especies y cinco géneros fúngicos bajo estudio, lo que demuestra que su espectro de acción es amplio.

La CH2, con menor espectro de acción, resultó ser efectiva frente a cuatro especies fúngicas incluidas en Alternaria sp., Phomopsis sp. y Fusarium sp., agentes causales de patologías en cultivos de relevancia agronómica y alimentaria.

\section{LITERATURA CITADA}

Agrios, G.N. 2005. Plant Pathology. p. 383615. $5^{\text {th }}$. ed. Academic Press, Burlington, Massachusetts, USA.

Alexopoulos, C.J., C.W. Mims, and M. Blackwell. 1996. Introductory Mycology. $4^{\circ}$ ed. 833 p. J W \& Sons Inc., California, USA.

Ali, H.K., A.M. Jumaah, and A.S. Hassian. 2017. Studying efficiency inhibition of some medicinal plant extracts against some fungal. ISSN: 2319-7706. International Journal of Current Microbiology and Applied Sciences 6:108-116. 
Araya González, P., C. Sandoval Briones, y F. Núñez Abarca. 2013. Eficiencia de distintos fungicidas sobre el control in vitro de Fusarium oxysporum aislado de frutos de kiwi. Tesis de Grado en Agronomía. Facultad de Ciencias Agrarias, Universidad de Talca, Chile. (Resumen).

Barnett, H., and B. Hunter. 1998. Illustrated genera of imperfect fungi. $3^{\circ}$ ed. 218 p. APS Press, St. Paul, Minnesota, USA.

Carpinella, M., L. Giorda, C. Ferrayoli, /and S. Palacios. 2003. Antifungal effects of different organic extracts from Melia azedarach L. on phytopathogenic fungi and their isolate active components. Journal of Agricultural and Food Chemistry 51:2506-2511.

Cavallo, A., R. Novo, y M. Perez. 2005. Eficiencia de fungicidas en el control de la flora fúngica transportada por semillas de maní (Arachis hypogaea) en la Argentina. Agriscientia 22:916.

Cesco, S., G. Neumann, N. Tomasi, R. Pinton, y L. Weisskopf. 2010. Liberación de flavonoides de origen vegetal en la rizosfera y su papel en la nutrición de las plantas. Planta y Suelo 329:1-25

CLSI. 2008. Reference method for broth dilution antifungal susceptibility testing of filamentous fungi; Approved. Standard. $2^{\text {nd }}$. ed. CLSI document M38-A2. Clinical and Laboratory Standards Institute, 950 West Valley Road, Suite 2500, Wayne, Pennsylvania, USA.

Desjardins, A. 2006. Fusarium Mycotoxins: Chemistry, Genetics and Biology. The American Phytopathological Society, St. Paul, Minnesota, USA.

Graham, Terrence, L. 1991. Flavonoid and isoflavonoid distribution in developing soybean seedling tissues and in seed and root exudates. Plant Physiol. 95:594-603.

Hernández, F., R. Pioli, A. Peruzzo, A. Formento, y G. Pratta. 2015. Caracterización morfológica y molecular de una colección de aislamientos de Phomopsis longicolla (teleomorfo desconocido: Diaporthales) de la región templada y subtropical de Argentina. Revista de Biología Tropical 63:871-884.

INTA. 2008. Aplicación eficiente de fitosanitarios. Instituto Nacional de Tecnología Agropecuaria (INTA). Ediciones INTA, Castelar, Buenos Aires, Argentina.

Iriti, M., y E.M. Varoni. 2017. Moviéndose al campo: inmunidad innata a las plantas en la protección de cultivos. International Journal of Molecular Sciences 18:640.
Leslie, J., and B. Summerell. 2006. The Fusarium Laboratory Manual. Blackwell Publishing, Iowa, USA.

Lygin, A.V., O.V. Zernova, C.B. Hill, N.A. Kholina, J.M. Widholm, G.L. Hartman, et al. 2013. Glyceollin is an important component of soybean plant defense against Phytophthora sojae and Macrophomina phaseolina. Phytopathology 103:984-994.

Lygin, A.V., C.B. Hill, M. Pawlowski, O.V. Zernova, J.M. Widholm, G.L. Hartman, et al. 2014. Inhibitory effects of stilbenes on the growth of three soybean pathogens in culture. Phytopathology 104:843-850.

Lozovaya, V.V., A.V. Lygin, S. Li, G.L. Hartman, and J.M. Widholm. 1999. Alteration of phenylpropanoid metabolism in soybean plants inoculated with Fusarium solani f. sp. glycines, the sudden death syndrome pathogen. En VI World Soybean Research Conference. 4-7 de Agosto. National Soybean Research Center, Chicago, USA.

Lozovaya, V., L. Anatoliy, O. Zernova, L. Shuxian, G. Hartman, and J. Widholm. 2004. Isoflavonoid accumulation in soybean hairy roots upon treatment with Fusarium solani. Plant Physiology and Biochemistry 42:671679.

Newton, A., C. Gravouil, y J. Fountaine. 2010. Gestión de la ecología de los patógenos foliares: tolerancia ecológica en los cultivos. doi:10.1111/j.1744-7348.2010.00437.x. Annals of Applied Biology 157:343-359.

Peruzzo, M.A., and R.N. Pioli. 2017. Effect of the toxicogenic ability of Fusarium graminearum on physiological quality of soybean seeds and flours. p. 119-152. In Soybean: cultivation, nutritional properties and effects on health. Nova Sc. Publishers, New York, USA.

Pioli, R., R. Benavídez, E. Morandi, y M. Bodrero. 2000. Estudio epidemiológico de patógenos fúngicos asociados a semillas y frutos de soja. Ed. Asoc. Latinoam. ISSN 0430-6155 Fitopatología 35:111-118.

Pioli, R.N., E.N. Morandi, M.C. Martínez, M.F. Lucca, A. Tozzini, V. Bisaro, et al. 2003. Morphological, molecular and pathogenic characterization of Diaporthe phaseolorum Variability in the core soybean producing area of Argentina. Am. Phytopathology Society Phytophology 93:136-146. 
Pioli, R., R. Hernández, M.L. Dietrich, C. Catalán, and E. Morandi. 2004. Isoflavonoids role in defense mechanisms associated with Glycine max - Diaporthe phaseolorum var. meridionalis interaction.VII World Soybean Research Conference- IV International Soybean Processing and Utilization Conference and III Congresso Mundial de Soja (Brazilian Soybean Congress). American Phytopathological Soc. Foz do Iguazú, PR, Brasil.

Rodríguez-Maturino, A, R. Troncoso-Rojas, A. Sánchez-Estrada, D. González-Mendoza, E. Ruiz-Sanchez, R. Zamora-Bustillos, et al. 2015. Efecto antifúngico de extractos fenólicos y de carotenoides de chilpetin (Caspicum anium var. glabriusculum) en Alternaria alternata y Fusarium oxysporum. Revista Argentina de Microbiología 47:72-77.

Sortino, M., M. Derita, y S. Zacchino. 2011. Diferentes estrategias para la búsqueda de compuestos antifúngicos. 231 p. Editorial Académica Española, Madrid, España.

Svetaz, L., A. Tapia, S. Lopez, R. Furlan, E. Petenatti, R. Pioli, et al. 2004. Antifungal chalcones and new caffeic acid esters from Zuccagnia punctata acting against soybean infecting fungi. Journal of Agricultural and Food Chemistry 52:3297-3300.
Taiz, L y E. Zeiger. 2006. Fisiología Vegetal. 1338 p. Capítulo 13. Publicaciones Universitat Jaume I. España.

Udayanga, D., P. Xingzhong Liu, W. Crous, E.H.C. McKenzie, E. Chukeatirote, and K.D. Hyde. 2012. A multi-locus phylogenetic evaluation of Diaporthe (Phomopsis). DOI 10.1007/s13225-012-0190-9. Fungal Diversity (2012) 56:157-171

Yanar, Y., I. Kadioğlu, A. Gökçe, I. Demirtaş, N. Gören, H. Çam, et al. 2011. In vitro antifungal activities of 26 plant extracts on mycelial growth of Phytophthora infestans (Mont.) de Bary. African Journal of Biotechnology 10:2625-2629.

Zacchino, S., y M. Gupta. 2007. Manual de técnicas in vitro para la detección de compuestos antifúngicos. Cap. 9 p. 85-97. Corpus Editorial y Distribuidora, Rosario, Santa Fe. Argentina 